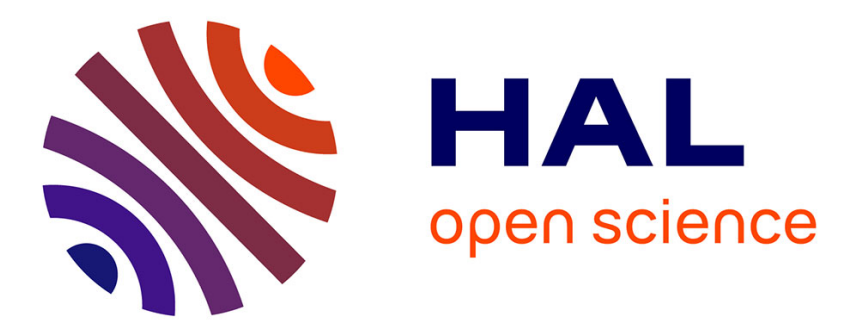

\title{
Two-dimensional Potential Fields for Advanced Implicit Modeling Operators
}

Loic Barthe, N A Dodgson, M A Sabin, B Wyvill, V Gaildrat

\section{To cite this version:}

Loic Barthe, N A Dodgson, M A Sabin, B Wyvill, V Gaildrat. Two-dimensional Potential Fields for Advanced Implicit Modeling Operators. Computer Graphics Forum, 2003, 22 (1), pp.23 - 33. 10.1111/1467-8659.t01-1-00643 . hal-01538469

\section{HAL Id: hal-01538469 https://hal.science/hal-01538469}

Submitted on 13 Jun 2017

HAL is a multi-disciplinary open access archive for the deposit and dissemination of scientific research documents, whether they are published or not. The documents may come from teaching and research institutions in France or abroad, or from public or private research centers.
L'archive ouverte pluridisciplinaire HAL, est destinée au dépôt et à la diffusion de documents scientifiques de niveau recherche, publiés ou non, émanant des établissements d'enseignement et de recherche français ou étrangers, des laboratoires publics ou privés. 


\title{
Two-dimensional potential fields for advanced implicit modeling operators
}

\author{
L. Barthe, N.A. Dodgson, M.A. Sabin, B. Wyvill and V. Gaildrat
}

University of Cambridge, University of Cambridge, Numerical Geometry ltd, University of Calgary, University of Toulouse

\begin{abstract}
Current methods for building models using implicit volume techniques present problems defining accurate and controllable blend shapes between implicit primitives. We present new methods to extend the freedom and controllability of implicit volume modeling. The main idea is to use a free-form curve to define the profile of the blend region between implicit primitives.

The use of a free-form implicit curve, controlled point-by-point in the Euclidean user space, allows us to group boolean composition operators with sharp transitions or smooth free-form transitions in a single modeling metaphor. This idea is generalized for the creation, sculpting and manipulation of volume objects, while providing the user with simplicity, controllability and freedom in implicit modeling.
\end{abstract}

Categories and Subject Descriptors (according to ACM CCS): I.3.5 [Computational Geometry and Object Modeling]: Curve, surface, solid, and object representations;

\section{Introduction}

Providing interactive, precise and intuitive control of shapes is a fundamental issue in the development of three dimensional modeling techniques. Direct manipulation of meshes, parametric shape representations and, more recently, subdivision surfaces are common and useful solutions adopted by most commercial software. Implicit volume models are rapidly becoming a practical alternative to these methods due to the increase in computing power and storage capacity of modern workstations combined with the latest developments in graphics hardware. Better hardware along with improved volume visualisation algorithms $\mathrm{s}^{1,2}$ and data structures $^{3}$, allow us to interactively and accurately render iso-potential surfaces or potential variations in an implicit volume.

In this paper, we denote as volume objects or implicit volumes three-dimensional objects which are defined by a potential field $f(p)$ that associates a potential value with each point $p$ of the Euclidean space $\mathbb{E}^{3}$. Commonly used surface representations such as sub-division surface techniques do not provide a true three-dimensional representation of the object. A surface in a volume representation is a set of points defined with an iso-potential value (an implicit surface). Vol- ume objects have several important advantages: inside and outside can be distinguished easily, they allow efficient collision tests, high quality triangle meshes of iso-potential surfaces $^{4}$, classical Boolean operations ${ }^{5}$, blending ${ }^{6,7,8}$, and more advanced sweeping by moving solid ${ }^{9}$, Boolean composition with soft transitions ${ }^{10,11}$ and Constructive Volume Geometry algebra ${ }^{12}$.

Most of these techniques are based on the blending properties of implicit surfaces. Early work used the addition operator between field values to provide smooth transitions (blends) between implicit primitives $6,13,14$. These transitions were approximately controlled by parameters embedded in the implicit function that defined these methods. Later work exploited the locality property of the primitives as a powerful method to build complicated objects from a small number of primitives combined with a large range of operators ${ }^{11,15}$. Composition operators like the ones proposed by Hoffmann et $\mathrm{al}^{16}$ and Pasko et $\mathrm{al}^{10}$ demonstrated that smooth transitions could be obtained using Boolean operators on volumes defined by the inequality $f(p) \leq 0$ (see also ${ }^{17}$ ), and Barthe et al ${ }^{18}$ showed that, in a restricted application, accurate control of the transition could be obtained. One of the big advantages over other modelling techniques is that a variety 
of composition operations can be easily performed between implicit primitives, thus accurate and intuitive control over these operations is a critical step to providing interactive and efficient volume modeling software.

The goal of this work is to provide methods that will simply and accurately control the transitions in composition operators and more generally increase the freedom in the manipulation of volume objects. We use the theoretical interpretation of composition operators described by Barthe et $\mathrm{al}^{18}$. This leads us from the definition of free-form implicit curves controlled point-by-point and with regular field variations, to the generalization of Boolean composition operators, sculpture and modeling tools in a unique operator based on the manipulation of this implicit free-form curve in the Euclidean modeling space. This greatly increases the simplicity, controllability and freedom in volume modeling.

The organization of this paper is as follows: we first present a summary of the different modeling techniques commonly used to build volume objects. Bounded primitives (such as "Soft Objects"14), real-functions and sampled potential field manipulations are described. It appears clear that, whatever the model used, improvements in control are desirable when volume primitives are composed.

This is followed by the presentation of free-form implicit curves and, more generally, their two-dimensional potential fields. Since these profiles are used to combine or define volumes, particular attention is focused on the variations of the field around the iso-potential curve. In this section, we present open and closed curves and we show the progress we have made in controlling the variations of the fields.

In Section 4 we present the application of free-form curves on volumes defined by the inequality $f(p) \leq 0$. This category regroups and generalizes most of the volume primitives, as shown by Adzhiev et al ${ }^{17}$. The possibility of creating open or closed free-form curves allows us to merge the Boolean composition operators (with or without smooth transitions) and operators to sculpt or create primitives in a single modeling metaphor: the extrusion of an implicit curve in an "implicit space". Whatever the operator, the user simply acts on the two combined potential functions $f_{1}$ and $f_{2}$ through the deformation of the implicit curve $G\left(f_{1}, f_{2}\right)=0$. We briefly illustrate the interactive manipulation of the implicit curves in a two-dimensional section of the Euclidean modeling space using the modeling tool introduced by Barthe et $\mathrm{al}^{19}$ and we discuss the limits of the intuitiveness and accuracy of this process.

\section{Related work}

Two different categories of potential function can be distinguished for modelling volume objects: the first form has functions which equal zero outside a boundary, and the second has functions which vary over the whole of space. This last form is more expensive in evaluation. Even though our paper only deals with non-bounded primitives, we briefly present both representations to allow the reader to clearly differentiate them.

\subsection{Bounded primitives}

"Metaballs"13 or "Soft Objects"14 are bounded objects defined by a potential function $f$ equalling zero everywhere outside the object's boundary. Inside this boundary, the potential varies from zero to one and the volume object is defined by the set of points of $\mathbb{E}^{3}$ for which $f(p) \geq C$ (where $C$ is a pre-chosen value in $] 0,1[$ ). A wide variety of primitives are available ${ }^{7,20}$, and the blend is automatically computed by summing the potential functions of the primitives. Many different blending functions ${ }^{21,22}$ and blending models ${ }^{23,24}$ have been proposed to control the smoothness of the transition region, but the operators remain limited to the blending and the control of which primitives must and must not blend. The locality of the definition and the capacity to be automatically blended allow modeling techniques based on these objects to be interactive ${ }^{25,26}$.

CSG composition operators are already supported by bounded primitives (using the Ricci's $\min / \max$ operators ${ }^{5}$ ) but $C^{1}$ discontinuities are introduced in the potential field of the resulting object, altering the smoothness of the transition when it has to be blended. This is undesirable.

A solution using Ricci's super-elliptic operator ${ }^{5}$ to apply binary union and binary intersection operators with smooth transitions to "Soft Objects" was used by Wyvill et al ${ }^{11}$ (see Equation 1), and extended to n-ary operations.

$$
G\left(f_{1}, f_{2}\right)=\left(f_{1}^{n}+f_{2}{ }^{n}\right)^{\frac{1}{n}}
$$

\subsection{Real functions and sampled potential fields}

R-functions (real functions) and sampled potential fields can be grouped into a second category ${ }^{17}$ where potentials are given over all the Euclidean space $\mathbb{E}^{3}$ and the volume object is defined by the inequality $f(p) \leq 0$. The opposite convention, where the volume is defined by $f(p) \geq 0$, can also be used. Bounded objects, described above, can be adapted to R-functions by considering only the distance field minus the radius of the primitive. Other primitives can be obtained from different sources when the potential field is reconstructed as a distance field ${ }^{27,28,29}$. These objects are fundamental for volume modeling, as much for the variety of primitives as for the generalization of volumes to a unified model. For R-function operators, volume objects are defined with the convention: $f(p) \geq 0$. It has been shown by Pasko et $\mathrm{al}^{10}$ how to apply binary CSG operators (with or without smooth transitions), space mapping operators and others. Equation 2 gives the example of the union operator with smooth transitions. 


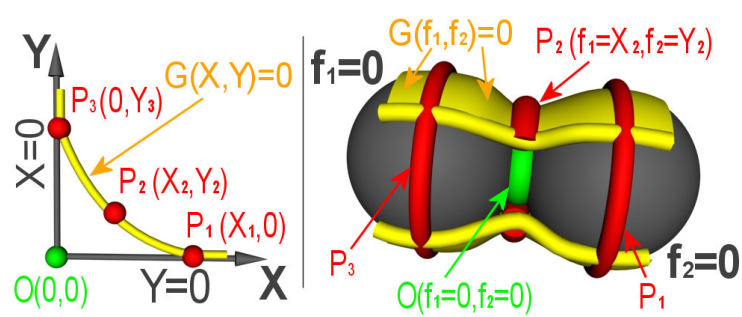

Figure 1: Representation of the same profile $G(X, Y)=0$ in $\mathbb{I}^{2}$ on the left and in $\mathbb{E}^{3}$ on the right, when $f_{1}$ and $f_{2}$ are two spherical potential fields. The resulting iso-potential surface (in yellow on the right) has been cut away to show the details underneath.

$$
G\left(f_{1}, f_{2}\right)=\left(f_{1}+f_{2}+\sqrt{f_{1}^{2}+f_{2}^{2}}\right)+\frac{a_{0}}{1+\left(\frac{f_{1}}{a_{1}}\right)^{2}+\left(\frac{f_{2}}{a_{2}}\right)^{2}}
$$

Parameters $a_{0}, a_{1}, a_{2}$ control the form of the transitions that do not have boundaries. Many other operators have been proposed in the literature ${ }^{16,30,8}$. While advanced operators, based on R-functions and displacement functions with local area of influence, allow the user to specify the location of the blend $^{31}$, the level of control on the transition itself remains globally equivalent. To correctly blend primitives, operator $G$ must satisfy specific properties that are well defined ${ }^{8}$. A binary operator $G$ applied on potential functions $f_{1}$ and $f_{2}$ can be written as a two-dimensional potential function $G(X, Y)$ composed with the combined three-dimensional potential functions $f_{1}$ and $f_{2}$ (Equation 3 ).

$$
\begin{aligned}
G: \mathbb{R}^{3} & \rightarrow \mathbb{R} \\
(x, y, z) & \rightarrow G\left(f_{1}(x, y, z), f_{2}(x, y, z)\right)
\end{aligned}
$$

Barthe et a ${ }^{18}$ introduced the notion of implicit extrusion fields. The function $G$ is considered as a zero iso-potential curve $G(X, Y)=0$ defined in a two-dimensional "implicit space" $\mathbb{I}^{2}$. A two-dimensional "implicit space" can be seen as a curvilinear space in which each coordinate is a potential field (see Section 2.3 and Equation 4).

$$
\begin{aligned}
G: \mathbb{I}^{2} & \rightarrow \mathbb{I} \\
(X, Y) & \rightarrow Z=G(X, Y) \\
\text { or }\left(f_{1}, f_{2}\right) & \rightarrow f_{3}=G\left(f_{1}, f_{2}\right) \\
\text { with } f_{i}: \mathbb{R}^{3} & \rightarrow \mathbb{R} \quad i=1 . .3 \\
(x, y, z) & \rightarrow f_{i}(x, y, z)
\end{aligned}
$$

To clarify notations, points of an "implicit space" are denoted with capital letters and points of the Euclidean space are denoted with small letters. A point $P(X=3, Y=2$ ) (or $\left.P\left(f_{1}=3, f_{2}=2\right)\right)$ in a space $\mathbb{I}^{2}$ is then represented in the Euclidean space $\mathbb{E}^{3}$ by the intersection between the 3 isopotential surface of the field defined by $f_{1}$ (which is the set of points $p$ of $\mathbb{E}^{3}$ for which $f_{1}(p)=3$ ) and the 2 iso-potential surface of the field defined by $f_{2}$ (which is the set of points $p$ of $\mathbb{E}^{3}$ for which $f_{2}(p)=2$ ). This is the intersection between two surfaces which is generally a curve. A point $P$ of $\mathbb{I}^{2}$ is represented by a curve in $\mathbb{E}^{3}$. A curve (or profile) can be seen as a continuous succession of points. The profile of $\mathbb{I}^{2}$ is then represented by a continuous succession of curves in $\mathbb{E}^{3}$ (representing each point of the profile), which gives us a surface. The profile $G(X, Y)=0$ defined in $\mathbb{I}^{2}$ is said to be extruded in $\mathbb{E}^{3}$ along the intersections of $f_{1}$ and $f_{2}$ iso-potential surfaces (Figure 1). The surface defined by the extrusion of profile $G=0$ is the result of combining $f_{1}$ and $f_{2}$.

Full details are given in ${ }^{18}$. The authors explain how points and vectors defining the profile in $\mathbb{I}^{2}$ can be directly selected by the user in Euclidean space $\mathbb{E}^{3}$, allowing accurate and intuitive control of the profile, and, therefore, of the resulting object. The profile $G=0$ is defined in ${ }^{18}$ by a function $H: \mathbb{R} \rightarrow \mathbb{R}$ such as $Y=H(X)$ and $G=Y-H(X)$. This greatly limits the freedom given to the user, and moreover removes a part of the intuitive process. It also obliges the authors to propose specific operators for the union, intersection and difference with a "functionally-defined" transition, which is a smooth transition defined point-by-point from the Euclidean space $\mathbb{E}^{3}$ with a single valued function $H: \mathbb{R} \rightarrow \mathbb{R}$. Functions $H$ are defined with one-dimensional cubic polynomial splines ${ }^{33}$ to interpolate the control points. Equation 5 shows the operator used for the union operator.

$$
G\left(f_{1}, f_{2}\right)=\min \left(f_{1}, f_{2}\right)-H\left(\left|f_{1}-f_{2}\right|\right)
$$

This union operator (first proposed by Dekkers et $\mathrm{al}^{32}$ ) is built with a min function which requires the $C^{1}$ continuity to be explicitly controlled where $f_{1}=f_{2}$. Our conclusion is that this model represents a very interesting theoretical base and more research has to be done on profile definition to exploit the properties of profile extrusion and point-by-point control from the Euclidean modeling space in a more powerful and intuitive volume modeling tool. To build a curve $G(X, Y)=0$ like the one shown in Figure 1, free-form implicit curves are needed (the vertical $X=0$ can not be defined with a single valued function). This leads us to the study of implicit freeform profiles controlled point-by-point.

\subsection{Definition of an implicit space}

An implicit space $\mathbb{I}^{n}$ is a space where each coordinate is a potential field. Its basis is defined by a set of $n$ linearly independent potential functions $f_{i}: \mathbb{E}^{m} \rightarrow \mathbb{E}$ and a point $P\left(X_{0}, . ., X_{n-1}\right)$ is expressed as $P\left(f_{0}=X_{0}, . ., f_{n-1}=X_{n-1}\right)$. In this paper $m=3$, hence each coordinate $f_{i}=X_{i}(i=$ $1 . . n-1)$ of a point $P$ is an iso-surface in $\mathbb{E}^{3}$ and the repre- 


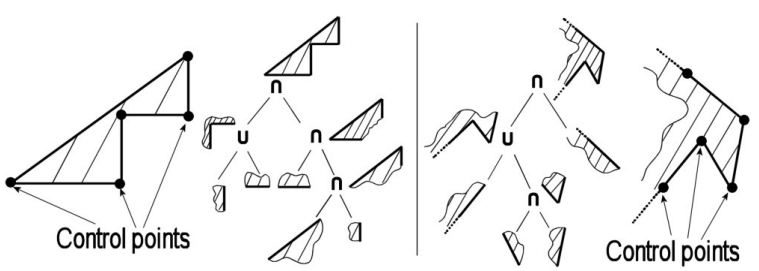

Figure 2: Example of binary composition trees to represent a polygon or an open curve by a real function.

sentation of the point $P$ itself is defined by their intersection. More details are given in ${ }^{18}$.

\section{Implicit free-form curves}

In this section we present our free-form implicit curves. We propose the use of homogeneous control parameters to provide an intuitive solution to the user and for this reason we use free-form implicit curves controlled point-by-point as a means of providing accurate and intuitive operators on volumes. The composition of two primitives gives a new object which can be used as a primitive in a new composition. Because the smoothness and the control of the form of the transition is highly dependent on the variational properties of the primitives' fields, potential fields used to combine them must preserve these properties as faithfully as possible. This is why particular attention has to be focused on the regularity of the field variations produced by our two-dimensional potential fields $G$.

\subsection{Natural solutions}

It appears natural to try to use methods like the implicitization of parametric free-form curves ${ }^{34,35}$ or the projection of the $z$ value of a surface defined by an equation $z=f(x, y)$ to define the implicit free-form curve. But these methods provide bounded functions while we need to be able to produce infinite open curves with the control of their limits. The first solution provides complicated equations and does not ensure regular and homogeneous potential field variations, and the second requires the user to design the entire surface to create the profile and its variations, while they should be ideally concentrating on the form of the curve. For these reasons we use a different approach.

\subsection{Free-form curves built with composition of lines}

As shown by Pasko et $\mathrm{al}^{36}$, it is possible to represent polygons with straight and curved edges by real functions. The polygon is decomposed in a binary composition tree where the leaves are lines and the nodes are union or intersection R-function operators (see Figure 2). This method also avoids internal and unwanted zeroes. We choose it as a starting point to create our profiles. Indeed, it can easily be adapted
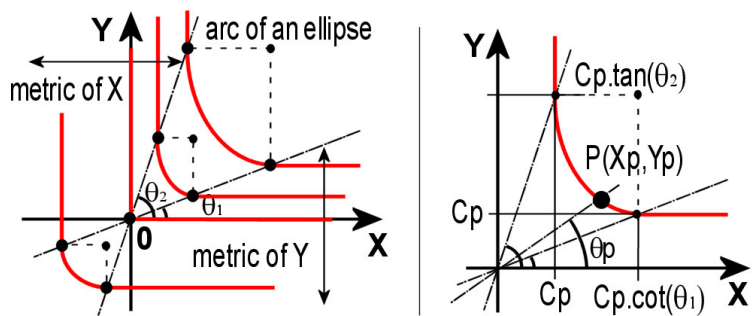

Figure 3: In red, on the left, representation of the twodimensional field iso-potential curves defining our $G^{1}$ continuous union operator $\widehat{G \cup}(X, Y)$. On the right, graph of the $C_{P}$ iso-potential curve and important values for computing the equation of the arc of an ellipse.

to open profiles, and because lines are combined, the extremities are perfectly controlled: they are simply half lines. To provide a regular base for the field variations, we use lines defined by the zero iso-potential of a linear potential function $l(X, Y)$. The linear potential function $l$ splits the space into two half spaces: one where $l(X, Y)<0$ (inside) and one where $l(X, Y)>0$ (outside). Function $l$ has the one isopotential line at a distance of one from the zero iso-potential (the one iso-potential line is in the outside half space defined by $l$, and the minus one iso-potential line in the inside half space). Because control points are used, line equations are defined with pairs of points. Even if R-function Boolean composition operators without smooth transition ${ }^{10}$ already provide a potential field that is $C^{1}$ continuous when both arguments are not equal to zero (they are both equal to zero at the junction between two zero iso-potential lines), they have a global impact on the line potential fields. This is a fundamental property because the variation of the combined primitives directly depends on the variation of the field of the composition operator and we want to provide volume objects with regular and smooth $C^{1}$ or at least $G^{1}$ continuous potential fields.

\subsection{Composition with better field variations}

To ensure more faithful conservation of the field variations, we propose a new operator that modifies line potentials only around their junction. Indeed, the line metric reproduces the metric of $X$ and $Y$ (as shown in Figure 3), i.e. the metric the potential functions $f_{1}$ and $f_{2}$ once used as a composition operator. For convenience, we propose the following terminology: $\widehat{G \cup}, \widehat{G_{\cap}}, \widehat{G_{\backslash}}$ represent respectively our $G^{1}$ continuous Boolean union, intersection and difference operators. Operator $\widehat{G \cup}(X, Y)$ (see Figure 3 and Equation 6) is defined by the fields of which it is composed outside a region bounded by two angles $\theta_{1}$ and $\theta_{2}$, and by an arc of an ellipse inside it. If the angles are close to one another, the field is sharp at the transition level and if $\theta_{1}$ is close to zero and $\theta_{2}$ close to $\pi / 2$, the field is highly smoothed. We suggest the use of 


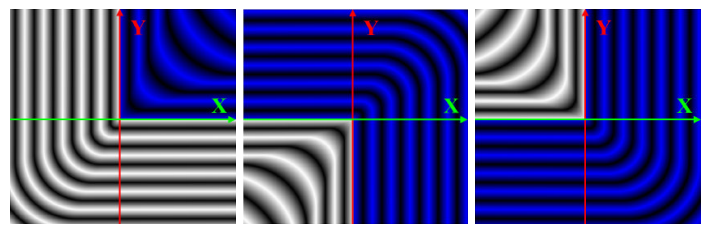

Figure 4: From left to right, graphs of our operators $\widehat{G \cup}$, $\widehat{G_{\cap}}$ and $\widehat{G_{\backslash}}$ in the space $\mathbb{I}^{2} . \widehat{G_{o p}} \geq 0$ in the blue area, and $\widehat{G_{o p}}<0$ in the white one.

$\theta_{1}=\pi / 8$ and $\theta_{2}=3 \pi / 8$, which gives a good average between a smooth field and the conservation of $X$ and $Y$ metrics.

$$
\left.\theta_{1} \in\right] 0, \pi / 4\left[, \quad \theta_{2} \in\right] \pi / 4, \pi / 2[
$$

At a point $P(X, Y): \theta=$ angle $([O X),[O P))$. We distinguish four distinct areas:

$$
\begin{aligned}
& P \in A_{1} \quad \text { if } \theta \in\left[\theta_{2}-\pi, \theta_{1}\right] \\
& P \in A_{2} \quad \text { if } \theta \in\left[\theta_{2}, \theta_{1}+\pi\right] \\
& \left.P \in A_{3} \quad \text { if } \theta \in\right] \theta_{1}, \theta_{2}[ \\
& \left.P \in A_{4} \quad \text { if } \theta \in\right] \theta_{1}+\pi, \theta_{2}+\pi[ \\
& \begin{cases}0 & \text { if } X=Y=0 \\
Y & \text { if } P \in A_{1} \\
X & \text { if } P \in A_{2} \\
C & \text { where } C \text { is the solution of: } \\
& \frac{\left(C . \cot \left(\theta_{1}\right)-X\right)^{2}}{\left(C . \cot \left(\theta_{1}\right)-C\right)^{2}}+\frac{\left(C . \tan \left(\theta_{2}\right)-Y\right)^{2}}{\left(C . \tan \left(\theta_{2}\right)-C\right)^{2}}=1 \\
& \text { if } P \in A_{3} \\
C & \text { where } C \text { is the solution of: } \\
& \frac{\left(X-C . \cot \left(\theta_{2}\right)\right)^{2}}{\left(C-C \cdot \cot \left(\theta_{2}\right)\right)^{2}}+\frac{\left(Y-C . \tan \left(\theta_{1}\right)\right)^{2}}{\left(C-C \cdot \tan \left(\theta_{1}\right)\right)^{2}}=1 \\
& \text { if } P \in A_{4}\end{cases}
\end{aligned}
$$$$
\begin{cases}0 & \text { if } X=Y=0 \\ Y & \text { if } P \in A_{1} \\ X & \text { if } P \in A_{2} \\ C & \text { where } C \text { is the solution of: }\end{cases}
$$$$
\widehat{G \cup}(X, Y)=\left\{\begin{aligned}
C & \text { where } C \text { is the solution of: } \\
& \frac{\left(C \cdot \cot \left(\theta_{1}\right)-X\right)^{2}}{\left(C \cdot \cot \left(\theta_{1}\right)-C\right)^{2}}+\frac{\left(C \cdot \tan \left(\theta_{2}\right)-Y\right)^{2}}{\left(C \cdot \tan \left(\theta_{2}\right)-C\right)^{2}}=1 \\
& \text { if } P \in A_{3}
\end{aligned}\right.
$$

Equation 6 appears, at first glance, to be difficult to solve, but it can be greatly optimized, and most of the terms can be pre-computed. The closed form solution for the evaluation of $C$ is given in Appendix B. Operators $\widehat{G_{\cap}}$ and $\widehat{G_{\backslash}}$ are built following the same construction and the same kind of equations are produced (their equations are given in Appendix A). These operators are real-valued binary CSG (Constructive Solid Geometry) operators, and their algebraic properties have been presented by Pasko et al ${ }^{10}$. However, we note that they are not symmetric $\left(\widehat{G_{o p}}(X, Y) \neq \widehat{G_{o p}}(Y, X)\right)$ if $\theta_{2} \neq$ $\frac{\pi}{2}-\theta_{1}$. With these operators, or with R-functions, free-form profiles, $G$, can be created. They are not smooth curves, but a succession of line segments, beginning and ending with a half line if they are open. To obtain smooth curves, we use the Boolean operators proposed in ${ }^{18}$. These operators provide a point-by-point control of a "functionally-defined" transition and, because they are derived from functions of $\mathbb{R} \rightarrow \mathbb{R}$, they provide regular and smooth field variations. The first point, the middle point and the last point of the
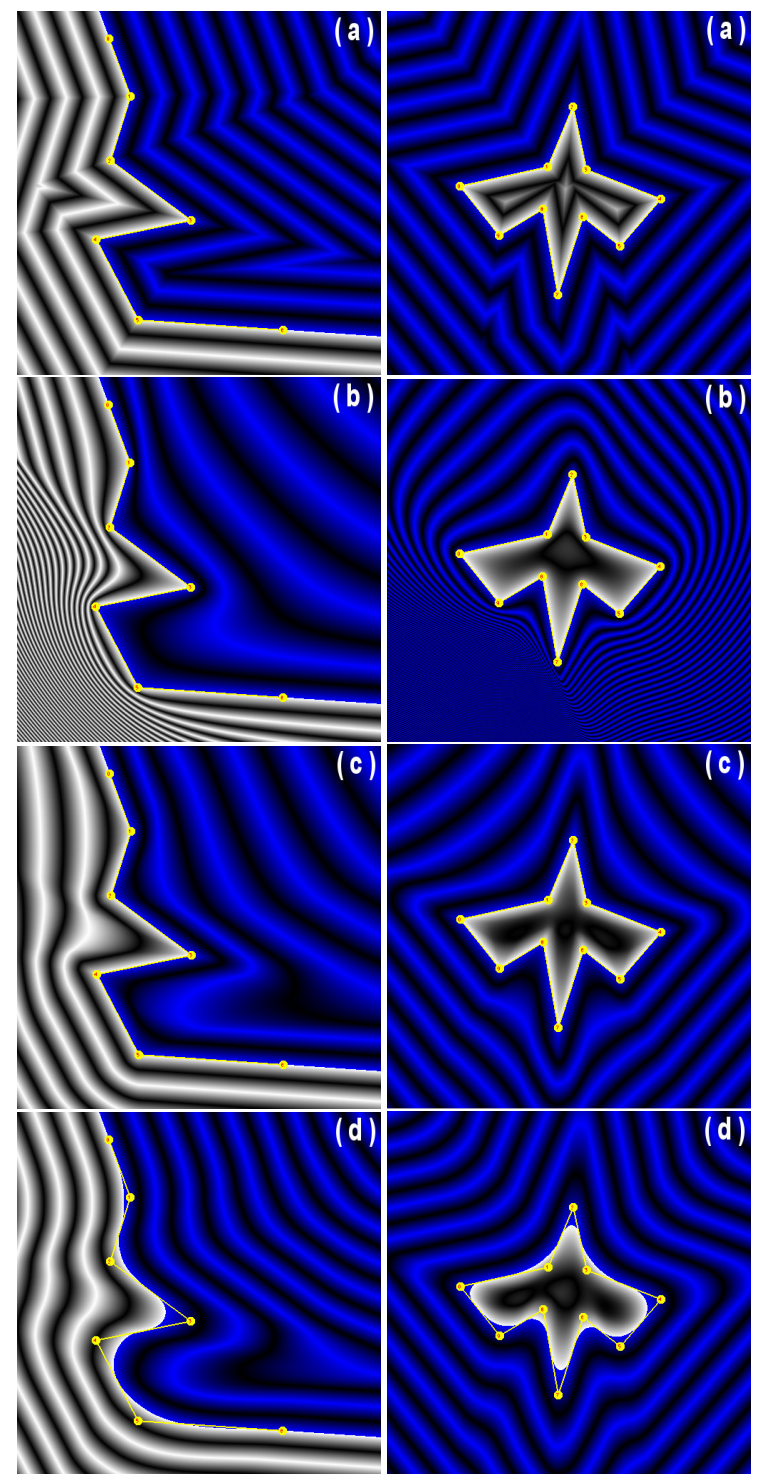

c)

Figure 5: Open and closed implicit free-form twodimensional potential fields $G$, shown in $\mathbb{E}^{2}$, built with different operators: (a) Ricci's min and max operators, (b) $R$ functions, (c) $\widehat{G \cup}$ and $\widehat{G_{\cap}}$, and (d) Barthe et al operators with smooth transitions. The white contours correspond to $G \leq 0$ and the blue ones to $G>0$. The control points and the corresponding lines are drawn in yellow. 


\begin{tabular}{ccccc}
\hline & min max & R-functions & $\widehat{G}$ & Barthe et al \\
\hline open & 106 & 148 & 457 & 135 \\
\hline closed & 178 & 254 & 802 & 227 \\
\hline
\end{tabular}

Table 1: Time in milliseconds to compute potential function values for the pictures shown in Figure 5. Picture size is $512^{2}$ pixels, which corresponds to 262144 evaluations. The open curves are built with six line segments and the closed curves are built with ten segments.

transition are then accurately controlled, which allows us to replace the sharp transition by a simple smooth transition automatically joining the middle of each segment. An exception is made for open curves where the beginning half-line is composed from the first control point and the last one is composed to the last point. The middle point is used to select the smoothness of the transition if necessary, but we recommend fixing it at a constant value to generate a smooth transition automatically and avoid the manipulation of an additional parameter.

\subsection{Results and discussion}

Figure 4 illustrates operators $\widehat{G \cup}, \widehat{G_{\cap}}$ and $\widehat{G_{\backslash}}$ individually and Figure 5 shows the difference of field variations obtained using different composition operators in both an open and a closed profile G. Ricci's min and max Boolean composition operators $^{5}$ leave the metric of the combined primitives unchanged (Figure 5(a)). This is why the potential field computed with the evaluation of the composition tree defining the profile $G$ with these operators gives a valid reference to evaluate the variations of the metric once the operators applied to compute the profile. Figure 5(b) shows how potential fields obtained using Pasko's R-function operators ${ }^{10}$ are degraded in some areas (bottom left corner for the open profile figure and bottom for the closed profile figure). As we see, our operators $\widehat{G \cup}$ and $\widehat{G_{\cap}}$ consequently increase the fidelity of the field variation for "segment profiles" (Figure 5(c)), and Barthe et al operators allow us to produce a smooth free-form implicit curve with regular and quite homogeneous variations in its potential field (Figure 5(d)). For these reasons, our profiles satisfy the essential properties to define combination operators on volume objects in efficient and controllable modeling tools. Computing times are given in Table 1 to compare the cost of the different operators used to create free-form profiles. We can note that the use of our new operators $\widehat{G}$ will increase the evaluation time of the curve by an average factor $\simeq 3.1$, while our smooth curves require less computations than the sharp ones produced with the R-function composition operators.

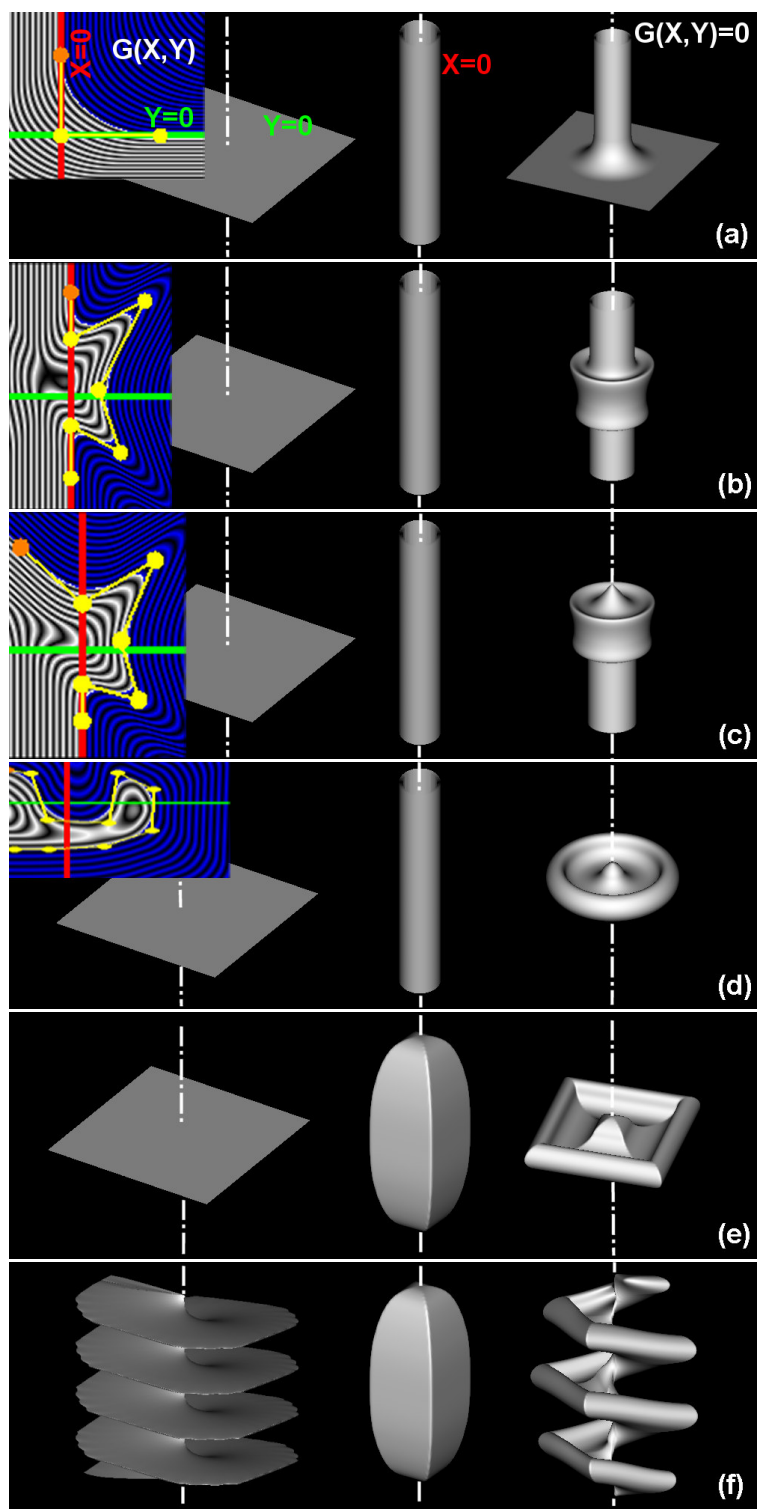

Figure 6: Figures (a) to (d) show different profiles extruded in an implicit field $\mathbb{I}^{2}$ defined by a cylindrical potential field (for the abscissa $X \equiv f_{1}$, in the central column) and a plane field (for the ordinate $Y \equiv f_{2}$, in the left column). Profiles are shown in the top left corner. They are extruded around the cylinder, following the horizontal direction given by the plane. The final object produced by the extrusion of the profile is shown in the right column. In Figures (e) and (f), the profile is the one used in Figure (d). Figure (e) illustrates the modification in the extrusion when the cylinder is replaced by a closed parallelepiped object, and Figure (f) illustrates the modification obtained when the plane is replaced by a screw-like object. The result in (f) is that the profile is extruded around the parallelepiped, following the iso-surfaces of the screw. 


\section{Extrusion of free-form curves}

In this section we show how our free-form profiles $G$ allow us to exploit the interpretation of "implicit space" to greatly increase freedom, accuracy and intuitive modeling in the manipulation of volumes. In fact, our method allows us to combine composition, sculpting and modeling tools in a single operator.

\subsection{Extrusion properties}

Following Barthe et $\mathrm{al}^{18}$, a two-dimensional function $G(X, Y)$ can be defined in an "implicit space" $\mathbb{I}^{2}$ to generate a volume object. The "implicit space" is defined by two potential functions of $\mathbb{E}^{3} \rightarrow \mathbb{E}, f_{1}$ and $f_{2}$. As shown in Figure 1 and explained in Section 2, the profile is said to be extruded along the intersections of $f_{1}$ and $f_{2}$ iso-potential surfaces. In effect, the choice of potential functions $f_{1}$ and $f_{2}$ define the trajectories of extrusion while the profile defines the free-from implicit curve which is going to be extruded along these trajectories (Figure 6).

The properties that a profile $G$ must respect in order to provide the union Boolean composition operator with smooth transitions are well known ${ }^{8}$, and "extrusion" properties of the profile are discussed in ${ }^{18}$. Briefly, when the profile follows the $X$ axis (which corresponds to the line $Y=0$ ), its representation in $\mathbb{E}^{3}$ follows the zero iso-potential surface of the field defined by $f_{2}$ and when it follows the $Y$ axis (which corresponds to the line $X=0$ ), its representation in $\mathbb{E}^{3}$ follows the zero iso-potential surface of the field defined by $f_{1}$ (see Figure 1). These properties allow us to integrate the zero iso-potential surfaces of primitives defined by $f_{1}$ and $f_{2}$, and to realize the Boolean composition operators with smooth transitions. Furthermore, as shown in Figure 7, in addition to the classical smooth transitions, the profile can be used to sculpt the primitives and to combine them with free-form transitions.

\subsection{Example of modeling interface}

The link between the profile and the shape of the resulting object becomes less intuitive when profiles are complicated. However, profile control points can be directly selected from the Euclidean space $\mathbb{E}^{3}$. Our volume objects are built and visualized using the modeling method proposed in ${ }^{19}$. We briefly summarize this approach to illustrate the controllability of our operators. Volumes are stored in a regular grid and visualized with a ray-casting rendering using a triquadratic reconstruction ${ }^{38}$. A plane section of the potential values is extracted from the grid and visualized as a picture in a new window (Figure 8 (a) and (b)). In this window, the user can interactively select the profile without the abstraction of the form of the iso-potential surfaces of potential fields $f_{1}$ and $f_{2}$ (Figure 8 (c) and (d)). This shows that being able to select the control points, and thus the profile directly in the Euclidean space $\mathbb{E}^{3}$, restores the accuracy lost by the definition of the

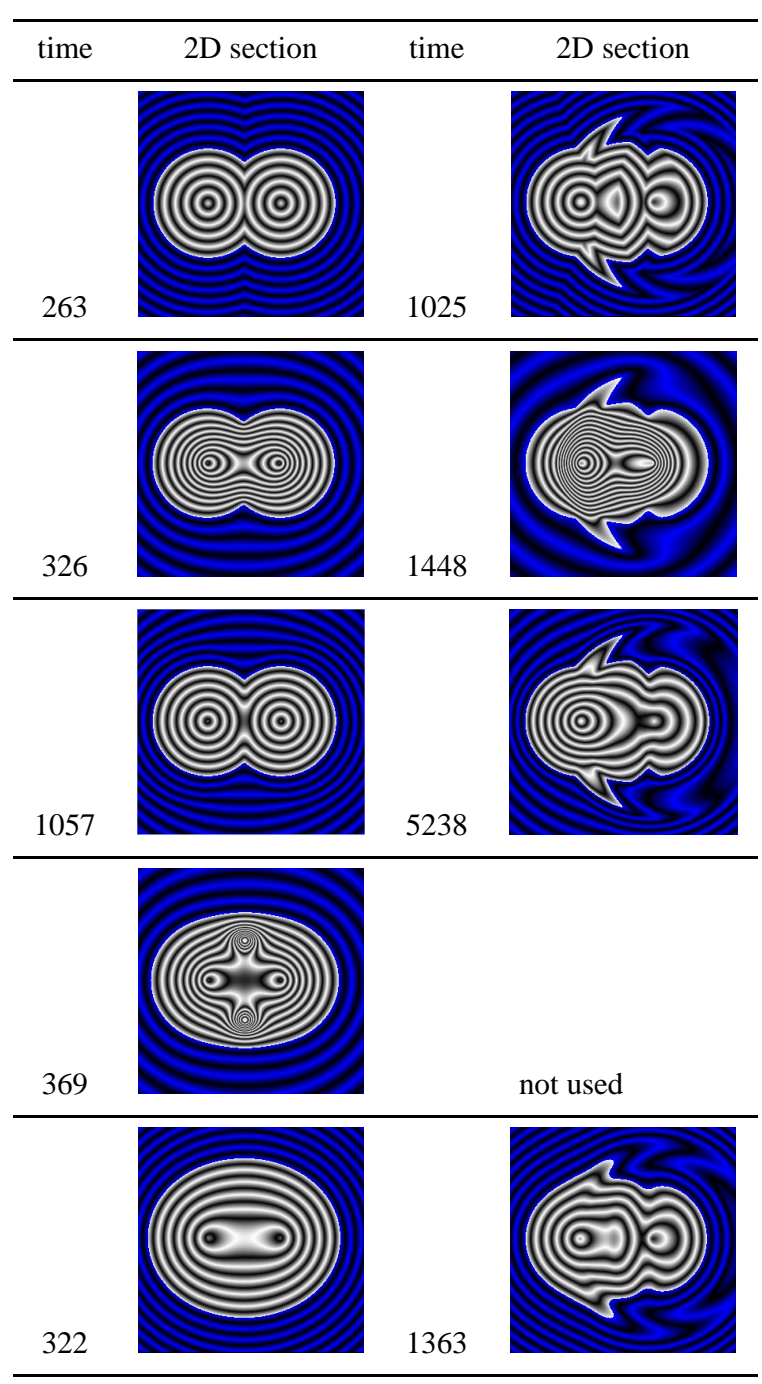

Table 2: Time in milliseconds to compute potential function values for a $128^{3}$ grid (2097152 evaluations). In the first column: $\left(1^{\text {st }}\right.$ row $)$ the Ricci's min max operators, $\left(2^{\text {nd }}\right.$ row $)$ the sharp $R$-functions operators, $\left(3^{\text {rd }}\right.$ row $)$ our operators $\widehat{G}$, $\left(4^{\text {th }}\right.$ row) the blending R-functions operators and $\left(5^{\text {th }}\right.$ row) our free-form curve operator. Time and potential field variations (in a two-dimensional plane section) are shown: On the left for the classical sharp/blending operators and on the right, for a free-form operator created with seven segments. 


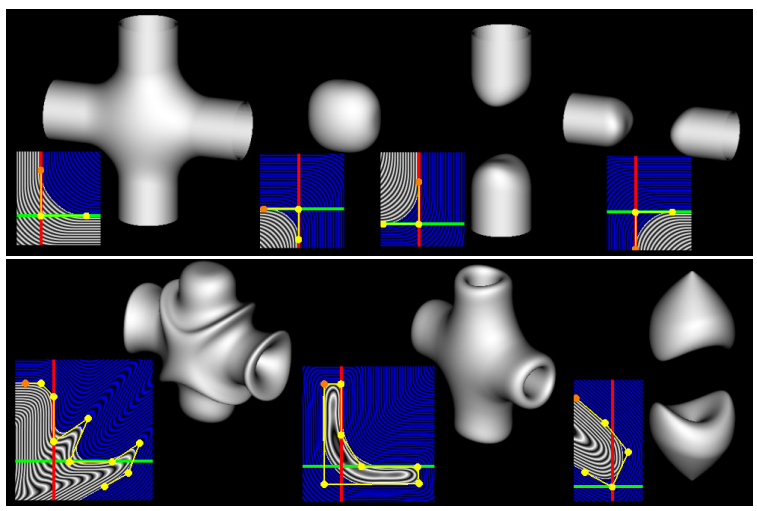

Figure 7: Different free-form profile extrusions in a "implicit space" defined by two orthogonal cylinders. The top row shows classical union, intersection and the two difference operators with smooth transitions. The following row illustrates more advanced possibilities offered by our freeform profile extrusion. In the profile pictures, the red line represents $X=0$ and the green line $Y=0$.

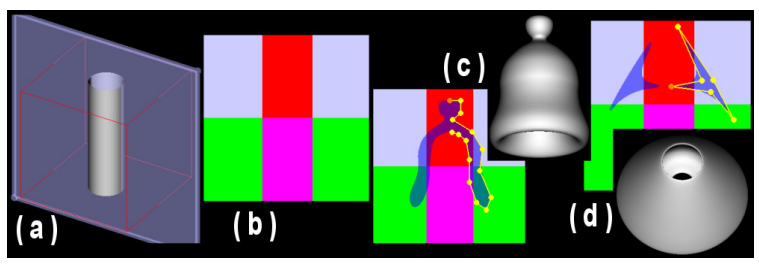

Figure 8: (a) Three dimensional visualisation of volume objects and selection of the plane section in transparent blue. (b) Visualisation of the plane section. Functions $f_{1}$ and $f_{2}$ are respectively: a vertical cylinder and an horizontal plane. Colors are used to easily identify different regions: red if $f_{1} \leq 0$ and $f_{2} \geq 0$, green if $f_{1} \geq 0$ and $f_{2} \leq 0$, purple if $f_{1}<0$ and $f_{2}<0$, bright gray if $f_{1}>0$ and $f_{2}>0$. (c) The section of the resulting object is visualized in blue with a transparency effect. We show an example of an open profile and its result. (d) Another example with a closed profile and its result.

profile in an "implicit space". Moreover, to exactly follow the zero iso-potential surface of potential function $f_{1}$ or $f_{2}$, the abscissa or the ordinate of a profile control points can be explicitly fixed to zero. This is important when profiles are used to combine primitives.

\subsection{Results and discussion}

With our approach, a wide variety of shapes and transitions can be produced, including all the ones that Barthe et al's models ${ }^{18}$ could generate (because free-form profiles generalize "functionally defined" ones). Furthermore, instead of disparate notions of composition operators, sculpture oper- ator or primitive creation tool, we present a unified modeling metaphor which is simply: choose the adequate potential functions $f_{1}$ and $f_{2}$ and create the profile $G$ to generate the new object. Once this process is well understood, our method is intuitive, and it provides a lot of freedom for the user to build different "implicit spaces" and extrude profiles in them (Figure 9). In addition, profiles can be directly defined and manipulated from the user modeling space $\mathbb{E}^{3}$. This makes our model relevant for interactive volume modeling. The limitations are essentially the complexity of the shape and the irregularity of the variations in the potential fields function $f_{1}$ and $f_{2}$. Table 2 illustrates the simple example of the composition of two spheres, the differences of computing time and the variations in the object potential field. Times correspond to the computation of the composition operator itself. In our application (briefly presented in the previous section), grids are used to store the potential fields after each operation. The given times correspond to the computation of a new $128^{3}$ voxels grid from the two grids representing the composed objects. Fast volume rendering methods based on ray-casting or hardware rendering can then perform the visualisation in about 1 second, and a basic marching-cube algorithm will extract the polygons from the grid in 5-10 seconds, depending on the object complexity (timings taken on an $866 \mathrm{MHz}$ AMD Athlon processor). If the object has to be visualized from its equation, these times illustrate the increasing of the computation complexity. The objects shown in Figure 9 have been built with a few primitives, and the average time to design one of them is about an hour and a half. Note that the twisted corkscrew's handle is generated by a profile swept and twisted along a line segment.

\section{Conclusion and future work}

Accurate and intuitive manipulation of implicit objects is the next required step to provide efficient implicit modeling software. In this paper we have presented some solutions to these requirements when implicit primitives are composed. Recent work introduced the notion of "implicit spaces" as a theoretical base to accurately control the transitions ${ }^{18}$ and we have shown how this leads us to the study of free-form implicit curves controlled point-by-point. Profiles require regular variations of their two-dimensional potential field and the control of their extremities. The adaptation of a method proposed by Pasko et $\mathrm{al}^{36}$ has allowed us to provide open and closed profiles, defining line segments or smooth curves, with sufficient properties.

To manipulate implicit volumes defined by the inequality $f(p) \leq 0$, we have grouped in a single modeling tool: creation, sculpture and Boolean composition operators with sharp or smooth free-form transitions. We have shown how accuracy is ensured and explained how to understand and use our modeling metaphor in an efficient way. The techniques described in this paper generalize the models given in 18 


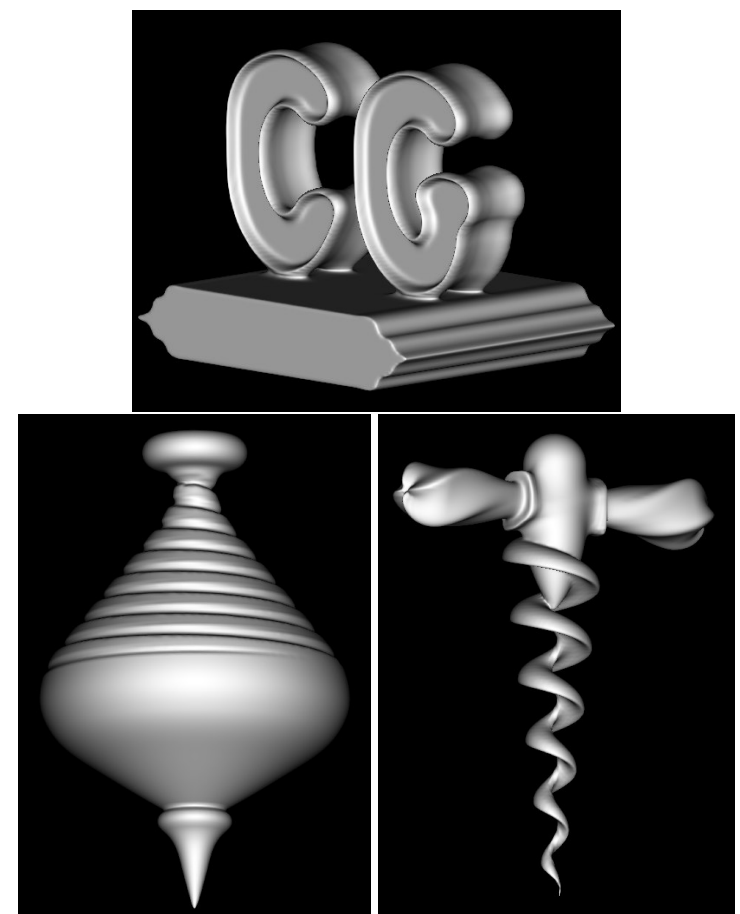

Figure 9: Examples of volume objects illustrating composition, sculpting and primitive creation using operators based on two-dimensional free-form potential fields.

and provide controllability and freedom of expression to the user and greatly extend the possibilities offered by operators on volumetric objects. The point-by-point control curve provides the possibility of interactively designing blended shapes, however more work needs to be done on volume data structure manipulation and modeling interface design to add interactivity to the modeling process. Adaptive structures like $\mathrm{ADF}^{3}$ and interactive ray casting algorithm for isosurface visualisation ${ }^{1}$ can be used to, respectively, store a sampled potential field and accurately render the surface. A sampled field structure stores the potential values after each operation, removing the expensive evaluation of an increasingly complicated potential field function. This technique allows us to accurately render the modeled object directly without the use of an additional data structure, such as a polygon mesh. These reasons provide a good justification for the investigation of the combination of these techniques, to provide interactive implicit modeling solutions.

Because sampled three-dimensional potential functions are large data structures, the development of multiresolution techniques to store and reconstruct potential fields represents another important step.

Free-form profiles can also be used in implicit sweep objects $^{20,37}$. Indeed, only profiles defined by single valued functions of $\mathbb{R} \rightarrow \mathbb{R}$ are used in implicit modeling, while there is no apparent reason to limit the user by the form of the profile they want to sweep. Our profiles should allow us to create swept objects defined by an inequality $f(p) \leq 0$. Since they have regular and homogeneous field variations, sweep objects can be correctly combined with other implicit objects.

\section{Acknowledgments}

This work was partially funded by E.U. contract HPRN-CT1999-00117

\section{References}

1. M. Chen, A. Kaufman and R. Yagel. Volume Graphics, Springer, London, 2000. 1, 9

2. K. Engel, M. Krauss and T. Ertl. High-quality Preintegrated Volume rendering Using Hardware-accelerated Pixel Shading. Proc. of EUROGRAPHICS/SIGGRAPH Graphics Hardware Workshop, 2001. 1

3. S.F. Frisken, R.N. Perry, A.P. Rockwood and T.R. Jones. Adaptatively Sampled Distance Fields: A General representation of Shape for Computer Graphics. Proceedings of SIGGRAPH 2000, Computer Graphics Proceedings, Annual Conference Series, pp. 249-254, 2000. 1,9

4. L.P. Kobbelt, M. Botsch, U. Schwanecke and H.P. Seidel. Feature Sensitive surface Extraction from Volume data. 1

Proceedings of SIGGRAPH 2001, Computer Graphics Proceedings, Annual Conference Series, pp. 57-66, 2001.

5. A. Ricci. A Constructive Geometry for Computer Graphics. The Computer Journal, 16 (2), pp. 157-160, 1973. $1,2,6$

6. J. F. Blinn. A Generalization of algebraic surface drawing. ACM Transaction on Graphics, 1 (3), pp. 235-256, 1982. 1

7. J. Bloomenthal and B. Wyvill. Interactive techniques for Implicit Modeling. Computer Graphics (Proc. of SIGGRAPH 1990), 24 (2), pp. 109-116, 1990. 1, 2

8. A. P. Rockwood. The Displacement Method for Implicit Blending Surfaces in Solid Models. ACM Transaction on Graphics, 8 (4), pp. 279-297, 1989. 1, 3, 7

9. A. Sourin and A. Pasko. Function Representation for Sweeping by a Moving Solid. IEEE Transaction on Visualization and Computer Graphics, 2 (1), pp. 11-18, 1996. 1

10. A. Pasko, V. Adzhiev, A. Sourin and V. Savchenko. 
Function Representation in Geometric Modeling: Concepts, Implementation and Applications. The Visual Computer, 8 (2), pp. 429-446, 1995. 1, 2, 4, 5, 6

11. B. Wyvill and A. Guy and E. Galin. Extending the CSG Tree: Warping, Blending and Boolean Operations in an Implicit Surface Modeling System. Computer Graphics Forum, 18 (2), pp. 149-158, 1999. 1, 2

12. M. Chen and J. Tucker. Constructive Volume Geometry. Computer Graphics Forum, 19 (4), pp. 281-293, 2000. 1

13. H. Nishimura, M. Hirai, T. Kawai, I. Shirakara and K. Omura. Object Modeling by Distribution Functions. Electronics Communications (in Japanese), J68 (D), pp. 718-725, 1985. 1, 2

14. G. Wyvill, C. McPheeters and B. Wyvill. Data Structure for Soft Objects. The Visual Computer, 2 (4), pp. 227234, 1986. 1,2

15. C. Galbraith, P. Prusinkiewicz and B. Wyvill. Modeling Murex Cabritii Sea Shell with a Structured Implicit Modeler. Proc. of Computer Graphics International 2000, 2000. 1

16. C. Hoffmann and J. Hopcroft. Automatic Surface Generation in Computer Aided Design. The Visual Computer, 1, pp. 92-100, 1985. 1, 3

17. V. Adzhiev, M. Kazakov and A. Pasko. Hybrid System Architechture for Volume Modeling. Computer and Graphics, 24 (1), pp. 67-78, 2000. 1, 2

18. L. Barthe, V. Gaildrat and R. Caubet. Extrusion of 1D implicit profiles: Theory and first application. International Journal of Shape Modeling, 7 (2), pp. 179-199, 2001. $1,2,3,4,5,7,8$

19. L. Barthe, B. Mora, N. Dodgson and M. Sabin. Triquadratic reconstruction for interactive modelling of potential fields. Proc. of Shape Modeling International 2002, pp. 145-153, 2002. 2, 7

20. B. Crespin, C. Blanc and C. Schlick. Implicit Sweep Objects. EUROGRAPHICS 1996, 15 (3), pp. 165-174, 1996. 2,9

21. Z. Kacic-Alesic and B. Wyvill. Controlled Blending of Procedural Implicit Surfaces. Proc. of Graphic Interface 1991, pp. 236-245, 1991. 2

22. Ca. Blanc and C. Schlick. Extended Field Functions for Soft Objects. Proc. of Implicit Surfaces 1995, pp. 21-32, 1995. 2

23. M. Desbrun and M.P. Gascuel. Animating Soft Substances with Implicit Surfaces. Computer Graphics (Proc. of SIGGRAPH 1995), pp. 287-290, 1995. 2

24. A. Guy and B. Wyvill. Controlled Blending for Implicit Surfaces Using a Graph. Proc. of Implicit Surfaces 1995, pp. 107-112, 1995. 2
25. E. Ferley and M. P. Cani and J. D. Gascuel. Practical Volumetric Sculpting. The Visual Computer, 16 (8), pp. 469-480, 2000. 2

26. E. Galin and S. Akkouche. Incremental Polygonization of Implicit Surfaces. Graphical Models, 62 (1), pp. 19$39,2000.2$

27. B.A. Payne and A.W. Toga. Distance Field Manipulation of Surface Models. Computer Graphics and Applications, 12 (1), pp. 65-71, 1992. 2

28. M. Jones and R. Satherley. Shape Representation Using Space Filled Sub-voxel Distance Fields. Proc. of Shape Modeling International 2001, pp. 316-325, 2001. 2

29. J.C. Carr, R.K. Beaton, J.B. Cherrie, T.J. Mitchell, W.R. Fright, B.C. McCallum and T.R. Evans. Reconstruction and Representation of 3D Objects with Radial Basis Functions. Proceedings of SIGGRAPH 2001, Computer Graphics Proceedings, Annual Conference Series, pp. 67-76, 2001. 2

30. A. E. Middleditch and K. H. Sears. Blend Surfaces for Set-theoretic Volume Modelling Systems. Computer Graphics, 19 (3), pp. 161-170, 1985. 3

31. G. Pasko, A. Pasko, M Ikeda and T. Kunii. Bounded Blending Operations. Proc. of Shape Modeling International 2002, pp. 95-103, 2002. 3

32. D. Dekkers, K. van Overveld and R. Golsteijn. Combining CSG Modeling with Soft Blending using Lipschitz-based Implicit Surfaces. Technical Report, Eindhoven University of Technology, Computer Graphics Group, 1997. 3

33. I.D. Faux and M.J. Pratt. Computational Geometry for Design and Manufacture, Ellis Horwood, 1979. 3

34. R.N. Goldman, T.W. Sederberg and D.C. Anderson. Vector Elimination: A Technique for the Implicitization, Inversion, and Intersection of Planar Parametric Rational Polynomial Curves. Computer Aided Geometric Design, 1 (4), pp. 327-356, 1984. 4

35. C.M. Hoffmann. Implicit Curves and Surfaces in CAGD. IEEE Computer Graphics and Applications, 13 (1), pp. 79-88, 1993. 4

36. A.A. Pasko, A.V. Savchenko and V.V. Savchenko. Implicit Curved Polygons. Technical Report 96-1-004, University of Aizu, Japon, 1996. 4, 8

37. C. Grimm. Implicit Generalized Cylinders Using Profile Curves. Proc. of Implicit Surfaces 1999, pp. 33-41, 1999. 9

38. B. Mora, J.P. Jessel and R. Caubet. Visualizaton of Isosurfaces with Parametric Cubes. EUROGRAPHICS 2001 Proc., 20 (3), pp. 377-384, 2001. 7 
Appendix A: Operators $\widehat{G_{\cap}}$ and $\widehat{G_{\backslash}}$

- Operator $\widehat{G_{\cap}}$ :

$\left.\theta_{1} \in\right] 0, \pi / 4\left[, \quad \theta_{2} \in\right] \pi / 4, \pi / 2[$. At a point $P(X, Y): \quad \theta=\operatorname{angle}([O X),[O P))$

We distinguish four distinct areas:

$$
\begin{array}{ll}
P \in A_{1} & \text { if } \theta \in\left[\theta_{2}-\pi, \theta_{1}\right] \\
P \in A_{2} & \text { if } \theta \in\left[\theta_{2}, \theta_{1}+\pi\right] \\
P \in A_{3} & \text { if } \theta \in] \theta_{1}, \theta_{2}[ \\
P \in A_{4} & \text { if } \theta \in] \theta_{1}+\pi, \theta_{2}+\pi[
\end{array}
$$

$$
\widehat{G \cup}(X, Y)=\left\{\begin{aligned}
0 & \text { if } X=Y=0 \\
X & \text { if } P \in A_{1} \\
Y \quad & \text { if } P \in A_{2} \\
C \quad & \text { where } C \text { is the solution of: } \\
& \frac{\left(X-C \cdot \cot \left(\theta_{2}\right)\right)^{2}}{\left(C-C \cdot \cot \left(\theta_{2}\right)\right)^{2}}+\frac{\left(Y-C \cdot \tan \left(\theta_{1}\right)\right)^{2}}{\left(C-C \cdot \tan \left(\theta_{1}\right)\right)^{2}}=1 \\
& \text { if } P \in A_{3} \\
C \quad & \text { where } C \text { is the solution of: } \\
& \frac{\left(C \cdot \cot \left(\theta_{1}\right)-X\right)^{2}}{\left(C \cdot \cot \left(\theta_{1}\right)-C\right)^{2}}+\frac{\left(C \cdot \tan \left(\theta_{2}\right)-Y\right)^{2}}{\left(C \cdot \tan \left(\theta_{2}\right)-C\right)^{2}}=1 \\
& \text { if } P \in A_{4}
\end{aligned}\right.
$$

- Operator $\widehat{G_{\backslash}}$ :

Operator $\widehat{G_{\searrow}}$ is directly obtained from operator $\widehat{G_{\cap}}$ using the following expression:

$$
\widehat{G_{\bigvee}}(X, Y)=\widehat{G_{\cap}}(X,-Y)
$$

\section{Appendix B: Closed form solution for the evaluation of} $C_{P}$ in our new composition operators

- Solution for the equation:

$$
\frac{\left(C \cdot \cot \left(\theta_{1}\right)-X\right)^{2}}{\left(C \cdot \cot \left(\theta_{1}\right)-C\right)^{2}}+\frac{\left(C \cdot \tan \left(\theta_{2}\right)-Y\right)^{2}}{\left(C \cdot \tan \left(\theta_{2}\right)-C\right)^{2}}=1
$$

$C$ is the greater solution of the following equation:

$$
\text { a. } C^{2}+b \cdot C+c=0
$$

with

$$
\begin{array}{r}
a=\frac{\left(\tan \left(\theta_{2}\right)-1\right)^{2}}{\tan ^{2}\left(\theta_{1}\right)}+\tan ^{2}\left(\theta_{2}\right) \cdot\left(\frac{1}{\tan \left(\theta_{1}\right)}-1\right)^{2} \\
-\left(\frac{1}{\tan \left(\theta_{1}\right)}-1\right)^{2} \cdot\left(\tan \left(\theta_{2}\right)-1\right)^{2} \\
b=-2 \cdot\left(X \cdot \frac{\left(\tan \left(\theta_{2}\right)-1\right)^{2}}{\tan \left(\theta_{1}\right)}+Y \cdot \tan \left(\theta_{2}\right) \cdot\left(\frac{1}{\tan \left(\theta_{1}\right)}-1\right)^{2}\right)^{2} \\
c=X^{2} \cdot\left(\tan \left(\theta_{2}\right)-1\right)^{2}+Y^{2} \cdot\left(\frac{1}{\tan \left(\theta_{1}\right)}-1\right)^{2}
\end{array}
$$

- Solution for the equation:

$$
\frac{\left(X-C \cdot \cot \left(\theta_{2}\right)\right)^{2}}{\left(C-C \cdot \cot \left(\theta_{2}\right)\right)^{2}}+\frac{\left(Y-C \cdot \tan \left(\theta_{1}\right)\right)^{2}}{\left(C-C \cdot \tan \left(\theta_{1}\right)\right)^{2}}=1
$$

$C$ is the lower solution of the following equation:

$$
\text { a. } C^{2}+b \cdot C+c=0
$$

with

$$
\begin{array}{r}
a=\frac{\left(\tan \left(\theta_{1}\right)-1\right)^{2}}{\tan ^{2}\left(\theta_{2}\right)}+\tan ^{2}\left(\theta_{1}\right) \cdot\left(\frac{1}{\tan \left(\theta_{2}\right)}-1\right)^{2} \\
-\left(\frac{1}{\tan \left(\theta_{2}\right)}-1\right)^{2} \cdot\left(\tan \left(\theta_{1}\right)-1\right)^{2} \\
b=-2 \cdot\left(X \cdot \frac{\left(\tan \left(\theta_{1}\right)-1\right)^{2}}{\tan \left(\theta_{2}\right)}+Y \cdot \tan \left(\theta_{1}\right) \cdot\left(\frac{1}{\tan \left(\theta_{2}\right)}-1\right)^{2}\right) \\
c=X^{2} \cdot\left(\tan \left(\theta_{1}\right)-1\right)^{2}+Y^{2} \cdot\left(\frac{1}{\tan \left(\theta_{2}\right)}-1\right)^{2}
\end{array}
$$

- All the terms in $\theta_{1}$ and $\theta_{2}$ can be precomputed (once $\theta_{1}$ and $\theta_{2}$ are selected), which greatly decreases the cost of the evaluation of $C$. For instance, $a$ can be totally precomputed. 